\title{
Response to vitamin D supplementation in different latitudes: results from two parallel placebo-controlled randomized controlled trials
}

\section{Abstract}

Vitamin D is vital to bone health and prolonged severe deficiency can lead to rickets in children and osteomalacia/osteoporosis in adults. Vitamin D is an exceptional nutrient in that its main source is exposure of the skin to UV rays, whilst it can also be ingested through diet. This study aimed to investigate the relative contribution of vitamin D supplementation and individual sunlight exposure in raising vitamin D levels, throughout winter, in ethnically identical adult women living in opposite latitudes. Within two parallel placebo-controlled randomized controlled trials $(\mathrm{RCT})$, with identical study designs, 135 Brazilian women, $($ England, $\mathrm{n}=56$, $51^{\circ} \mathrm{N}$; Brazil, $\mathrm{n}=79,16^{\circ} \mathrm{S}$ ), were randomized to receive daily $15 \mu \mathrm{g}$ vitamin D3 supplements or placebo, for 12 weeks. Oral vitamin $\mathrm{D}$ supplementation of $15 \mu \mathrm{g}$ daily was effective at raising 25-hydroxivitamin D [25(OH)D] concentrations over winter, regardless of latitude, and the response was dependent on baseline vitamin D status. In both latitudes, supplementation prevented the seasonal concomitant increase in plasma parathyroid hormone (PTH) levels. Additionally, the individual UV radiation level was strongly correlated with $25(\mathrm{OH}) \mathrm{D}$ concentrations. The research also showed: 1) an optimal vitamin D status for bone health around 70-80 nmol/l; 2) the required UV radiation to achieve this status was $1.5 \mathrm{SED} ; 3$ ) the vitamin D dietary intakes required to achieve these serum levels were $4.5 \mu \mathrm{g} / \mathrm{d}$ at a low latitude $\left(16^{\circ} \mathrm{S}\right)$ and $37 \mu \mathrm{g} / \mathrm{d}$ at high latitude $\left(51^{\circ} \mathrm{N}\right)$. The strength of these results is the novel analysis that directly links human in vivo individual sunlight radiation, increased vitamin $\mathrm{D}$ intake and $25(\mathrm{OH}) \mathrm{D}$ status, within two parallel RCTs in opposite latitudes. This study demonstrated that a daily supplement of $15 \mu \mathrm{g}$ vitamin $\mathrm{D}_{3}$ was an effective strategy to significantly raise vitamin D status throughout the winter months in adult females, with important implications for bone health through the simultaneous lowering of PTH, regardless of latitude. Our data gives a two-fold contribution to the vitamin D field: firstly it will help raise awareness of the risk for vitamin D deficiency in low latitude regions such as most countries in South America as well as amongst South-Americans living in higher latitudes, particularly in the UK; secondly it provides key data for setting appropriate vitamin D recommendations for Brazil (which currently follows US recommendations) as well as for similar latitudes.

\section{Conflict of Interest}

There is no conflict of interest. 